\title{
Sperm Retrieval Techniques for Assisted Reproduction
}

\author{
Sandro C. Esteves, Ricardo Miyaoka, Ashok Agarwal \\ ANDROFERT, Andrology and Human Reproduction Clinic (SCE, RM), Campinas, São Paulo, Brazil \\ and Center for Reproductive Medicine, Glickman Urological and Kidney Institute (AA), Cleveland \\ Clinic, Cleveland, Ohio, USA
}

\begin{abstract}
Different surgical methods such as PESA, MESA, TESA, TESE and micro-TESE have been developed to retrieve spermatozoa from either the epididymis or the testis according to the type of azoospermia, i.e., obstructive or non-obstructive. Laboratory techniques are used to remove contaminants, cellular debris, and red blood cells following collection of the epididymal fluid or testicular tissue. Surgically-retrieved spermatozoa may be used for intracytoplasmic sperm injection (ICSI) and/or cryopreservation. In this article, we review the surgical procedures for retrieving spermatozoa from both the epididymis and the testicle and provide technical details of the commonly used methods. A critical analysis of the advantages and limitations of the current surgical methods to retrieve sperm from males with obstructive and non-obstructive azoospermia is presented along with an overview of the laboratory techniques routinely used to process surgically-retrieved sperm. Lastly, we summarize the results from the current literature of sperm retrieval, as well as the clinical outcome of ICSI in the clinical scenario of obstructive and nonobstructive azoospermia.
\end{abstract}

Key words: infertility; male; azoospermia; sperm retrieval; reproductive techniques; assisted; review Int Braz J Urol. 2011; 37: 570-583

\section{INTRODUCTION}

Within the last decades there were two major achievements in the area of male infertility (1-3). The first was the introduction of intracytoplasmic sperm injection (ICSI) for the treatment of male factor infertility due to severely abnormal semen quality (1). The second was the extension of ICSI to azoospermic males and the demonstration that spermatozoa retrieved from either the epididymis or the testis were capable of normal fertilization and pregnancy $(2,3)$. Azoospermia, defined as the complete absence of spermatozoa in the ejaculate after centrifugation, is found in $1-3 \%$ of the male population and in approximately $10 \%$ of the infertile males. Although azoospermia is associated with infertility, it does not necessarily imply sterility because many azoospermic men maintain sperm production at varying levels within the testes (4). Several sperm retrieval methods have been developed to collect sperm from the epididymis or the testis of azoospermic men. Surgically-retrieved spermatozoa can be used to induce pregnancy through assisted reproductive techniques (ART), i.e., in vitro fertilization associated to ICSI (1-7).

In this article, we review the methods for retrieval of epididymal and testicular spermatozoa and their success rates in different clinical conditions. We provide a critical appraisal of the advantages and limitations of the current surgical methods to retrieve sperm from male patients with obstructive (OA) and non-obstructive azoospermia (NOA). The reproductive potential of the male gamete extracted from the epididymis or the testis, and used for assisted fertilitization is also reviewed. 
Evaluation of azoospermic patients prior to sperm retrieval.

The choice of sperm retrieval technique and its success rate is dependent on the type of azoospermia (obstructive or non-obstructive). Clinical history, physical examination and laboratory tests for endocrine assessment (serum follicle-stimulating hormone $[\mathrm{FSH}]$ and testosterone levels) are useful pre-operative diagnostic tools. Together, these factors provide a $\sim 90 \%$ prediction of the azoospermia type (5). In OA spermatogenesis is intact but a mechanical blockage exists somewhere between the epididymis and the ejaculatory duct. Acquired OA include vasectomy, failure of vasectomy reversal, post-infectious diseases, surgical procedures in the scrotal, inguinal, pelvic or abdominal regions, and trauma $(4,6)$. Congenital causes of OA include cystic fibrosis, congenital absence of the vas deferens (CAVD), ejaculatory duct or prostatic cysts and Young's syndrome (4-7). Nonobstructive azoospermia comprises a spectrum of testicular histopathology patterns resulting from various causes that include environmental toxins, medications, genetic and congenital abnormalities, varicocele, trauma, endocrine disorders, and idiopathic (4-7).

Men with OA usually have normal sized testes and hormone profile. Occasionally, the epididymis or the seminal vesicles may be enlarged or a cyst can be palpable on rectal examination. The presence of a low volume $(<1.5 \mathrm{~mL})$ acidic $(\mathrm{pH}$ $<7.0$ ) azoospermic ejaculate, with absent or low fructose and epididymal thickening, associated to nonpalpable vasa deferentia is pathognomonic of OA $(4,6)$. Approximately two-thirds of men with OA and CAVD have mutations of the cystic fibrosis transmembrane conductance regulator (CFTR) gene. Failure to identify a CFTR abnormality in a man with CAVD does not rule out the presence of a mutation, since some are undetectable by routine testing methods. The female partner should be offered CF testing before proceeding with treatments that utilize the sperm because of the high risk of the female being a CF carrier. If a CFTR gene mutation is identified ( $\sim 4 \%$ of female partners are carriers), counseling is recommended before proceeding with sperm retrieval and ICSI due to the risk of the transmission of cystic fibrosis to the offspring $(4,6-9)$.
Azoospermic men with idiopathic obstruction and men with a clinical triad of chronic sinusitis, bronchiectasis, and obstructive azoospermia (Young's syndrome) may be at higher risk for $\mathrm{CF}$ gene mutations as well. In such cases, testing for CF mutations and counseling is also advisable $(4,6)$.

Men with FSH levels, testicular size and ejaculate volume within normal ranges may have either NOA or OA $(4,6-9)$. In such cases, a testicular biopsy may be required to provide a definitive diagnosis. Histopathological evaluation of testicular specimens indicates the presence of normal spermatogenesis in cases of OA while hypospermatogenesis or maturation arrest or Sertoli cell-only (SCO) are seen in men with NOA. A testicular biopsy may be dismissed in cases of elevated FSH and small testes because this association is indicative of NOA $(4,7)$. However, a biopsy may be considered to determine the likelihood of sperm retrieval in ICSI candidates with NOA. The presence of either spermatozoa on a wet prep or hypospermatogenesis on testicular histopathology is highly predictive of successful sperm retrieval in future retrieval attempts $(8,10)$. Conversely, the absence of sperm in a biopsy specimen does not absolutely exclude the chances of finding sperm elsewhere within the testis due to the heterogenic distribution of spermatogenesis in NOA men $(5,8,10)$.

Karyotyping and Y-chromosome microdeletion testing should be offered to men with NOA of unknown origin. Karyotypic abnormalities affect $10-15 \%$ of men with NOA, and the Klinefelter syndrome (KS) accounts for approximately two-thirds of the cases $(8,11)$. Y-chromosome infertility is seen in $7-15 \%$ of men presenting with NOA. Genetic testing may provide prognostic information for sperm retrieval (5-8). Azoospermic patients with Y-chromosome microdeletions restricted to the AZFc region may harbor viable sperm within the testis. In contrast, the chances of finding sperm in men with complete AZFa or AZFb deletions is virtually zero $(12,13)$.

\section{WHAT ARE THE AVAILABLE SPERM RE- TRIEVAL TECHNIQUES?}

In Table 1 we summarize the commonly used methods to retrieve sperm and their indications. 
Table 1 - Sperm Retrieval Techniques and their Indications for Assisted Reproduction.

\begin{tabular}{|c|c|c|}
\hline Technique & Acronym & Indications \\
\hline Percutaneous epididymal sperm aspiration & PESA & OA cases only \\
\hline Microsurgical epididymal sperm aspiration & MESA & OA cases only \\
\hline Testicular sperm aspiration & TESA; TEFNA $^{1}$ & $\begin{array}{c}\text { Failed PESA in OA } \\
\text { Epididymal agenesis in CAVD cases } \\
\text { Favorable testicular histopathology }{ }^{2} \\
\text { in NOA } \\
\text { Previous successful TESA attempt } \\
\text { in NOA }\end{array}$ \\
\hline $\begin{array}{l}\text { Testicular sperm extraction (single or } \\
\text { multiple biopsies) }\end{array}$ & TESE & $\begin{array}{c}\text { Failed PESA or TESA in OA } \\
\text { NOA cases }\end{array}$ \\
\hline Microsurgical testicular sperm extraction & Micro-TESE & NOA cases only \\
\hline
\end{tabular}

OA: obstructive azoospermia; NOA: nonobstructive azoospermia

1 - Testicular fine-needle aspiration (TEFNA) is a technical variation of TESA; 2 - Hypospermatogenesis

\section{Percutaneous Sperm Retrieval Methods}

Craft and Shrivastav, in 1994, first described the use of the percutaneous approach to retrieve sperm from the epididymis (14). Two years later, Lewin et al. reported the use of testicular fine needle aspiration to retrieve sperm from the testis (15). Percutaneous retrievals are usually undertaken under local anesthesia only or in association with intravenous sedation. Percutaneous sperm retrieval can be either diagnostic or therapeutic. In the former, it is used to confirm the presence of viable spermatozoa prior to ICSI. In the latter, it is carried out at the same day of oocyte retrieval or at the day before.

\section{Percutaneous Epididymal Sperm Aspiration (PESA)}

For PESA, a fine needle (e.g, 26 gauge) attached to a $1 \mathrm{~mL}$ tuberculin syringe is inserted through the scrotal skin into the epididymis (Figure-1A). Negative pressure is created by pulling the syringe plunger while the tip of the needle is gently moved in and out inside the epididymis until a clear fluid is seen coming into the syringe. The amount of fluid aspirated is often minimal $(\sim 0.1 \mathrm{~mL})$, except in cases of CAVD in which $0.3-1.0 \mathrm{~mL}$ may be obtained. The aspirate is flushed into a tube containing warm sperm medium. The tube containing the epididymal aspirate is taken to the laboratory for immediate microscopic examination (Figure-1C). PESA is repeated at a different site (from cauda to caput epididymis) until adequate number of motile sperm is retrieved. If PESA fails to retrieve motile sperm, testicular sperm retrieval can be attempted at the same operative time.

\section{Testicular Sperm Aspiration (TESA)}

Despite minor technical variations, the common principle of all methods described for TESA involves the needle insertion through the scrotal skin 


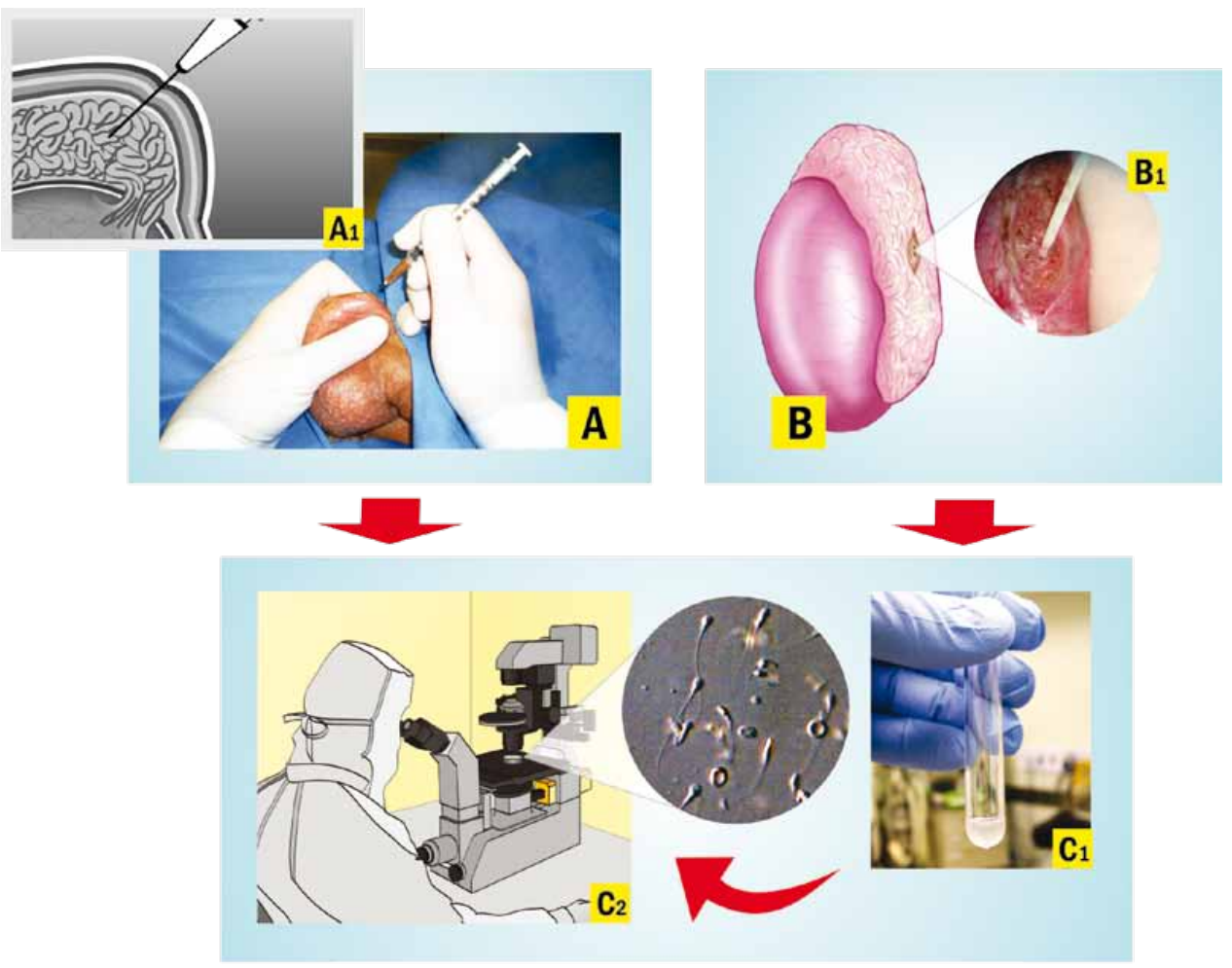

ANDROFERT 2011

Figure 1 - Epididymal Sperm Retrieval Techniques. A) Percutaneous Epididymal Sperm Aspiration (PESA). Epididymis is stabilized between the index finger, thumb and forefinger. A needle attached to a tuberculin syringe is inserted into the epididymis through the scrotal skin (A1), and fluid is aspirated. B) Microsurgical Epididymal Sperm Aspiration (MESA). After epididymis exposure, a dilated epididymal tubule is microdissected and opened. Fluid emanating from the epididymal tubule is aspirated using an angiocatheter attached to a tuberculin syringe (B1). C) Laboratory Sperm Handling. Aspirates are flushed into a tube containing HEPES-buffered sperm medium and sent for microscope examination (C1). Aspirate aliquots are spread onto a Petri dish and are examined microscopically to confirm the presence of motile sperm (C2).

into the testis. Then, testicular parenchyma is percutaneously aspirated using fine (e.g. 22 gauge) or large diameter needle (e.g., 18 gauge). The needle is usually inserted at the anteromedial or anterolateral portion of the superior testicular pole, in an oblique angle towards the medium and lower poles. These areas are least likely to contain major branches of the testicular artery running superficially underneath the albuginea. Loupe-magnification may be used to avoid small vessels seen through the skin. Negative pressure is created by pulling the syringe plunger while the tip of the needle is moved in and out the testis in an oblique plane to disrupt the seminiferous tubules and sample diffe- rent areas (Figure-2A). The specimen is flushed into a tube containing warm sperm medium, and is immediately transferred to the laboratory for microscopic examination (Figure-2C). TESA or TESE may be performed at the contralateral testis if insufficient or no sperm are obtained.

\section{Microsurgical Sperm Retrieval Techniques}

Microsurgical sperm retrieval can be performed under either local anesthesia in association with intravenous sedation or epidural anesthesia. Operating microscope and microsurgery techniques are used throughout the procedures. 


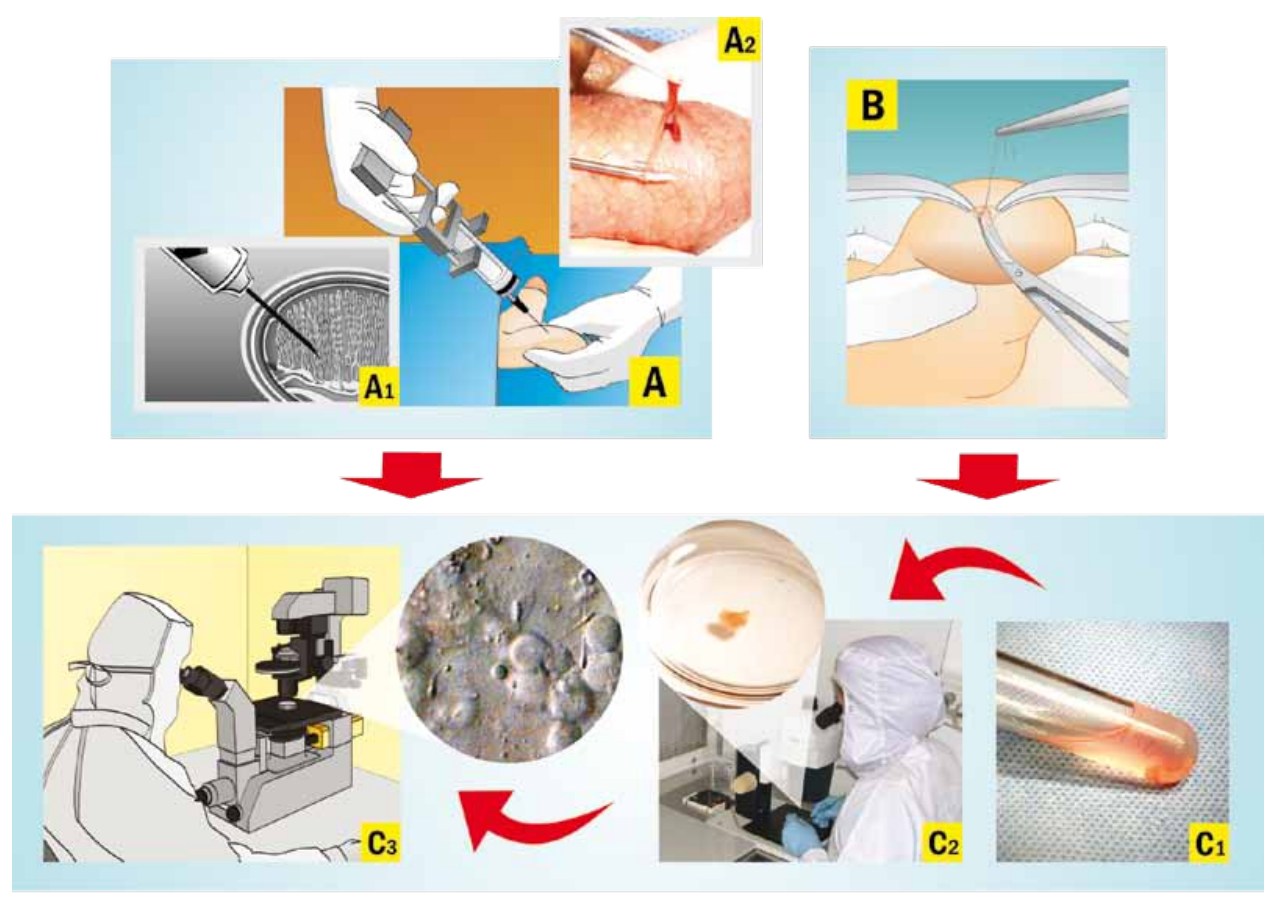

ANDROFERT 2011

Figure 2 - Testicular Sperm Retrieval Techniques. A) Percutaneous Testicular Sperm Aspiration (TESA). A needle attached to a syringe is percutaneous inserted into the testis. Negative pressure is created and the tip of the needle is moved within the testis to disrupt the seminiferous tubules and sample different areas (A1). A piece of testicular tissue is aspirated, and a forceps is used to remove the seminiferous tubules that exteriorize from the scrotal skin (A2). B) Conventional Testicular Sperm Extraction (TESE). A 1-2 cm skin incision is made to allow opening of scrotal layers down to the albuginea. Testicle is not exteriorized from scrotum. A small incision is made in an avascular area of the albuginea to expose testicular parenchyma. A fragment of approximately $5 \times 5 \mathrm{~mm}$ is excised. Additional fragments may be taken from the same incision or from different testicular poles using multiple incisions. C) Laboratory Sperm Handling. Testicular specimens are flushed into a tube containing sperm medium (C1), and the tube is transferred to the laboratory for tissue processing. Testicular fragments are washed-free from blood clots, and seminiferous tubules are mechanically dispersed using needled-tuberculin syringes until no intact tubules are seen (C2). Testicular homogenates are microscopically examined to confirm the presence of spermatozoa (C3).

\section{Microsurgical Epididymal Sperm Aspiration (MESA)}

MESA was first described by Temple-Smith et al. in 1985 (16). The surgical technique involves the exteriorization of the testis through a $2-3 \mathrm{~cm}$ transverse scrotal incision. The epididymal tunica is incised and an enlarged tubule is then dissected and opened with sharp microsurgical scissors. Fluid exuding from the tubule is aspirated with the aid of a silicone tube or blunted needle attached to a tuberculin syringe (Figure-1B). The aspirate is flushed into a tube containing warm sperm medium and is transferred to the laboratory for examination (Figure-1C). MESA is repeated at a different site of the same epididymis (from cauda to caput) and/or at the contralateral one until adequate number of motile sperm is retrieved. If MESA fails to retrieve motile sperm, TESA or TESE can be performed at the same operative time.

\section{Microsurgical Testicular Sperm Extraction (mi- cro-TESE)}

Microsurgical-guided testicular sperm extraction was originally described by Schlegel in 1999 (17). The delivery of the testis is carried out as des- 
cribed for MESA. Then, a single, large, mid-portion incision is made in an avascular area of the tunica albuginea under 6-8x magnification and the testicular parenchyma is widely exposed (Figure-3). Dissection of the testicular parenchyma is undertaken at $16-25 \mathrm{x}$ magnification searching for enlarged islets of seminiferous tubules (more likely to contain germ cells and eventually normal sperm production). The superficial and deep testicular regions may be examined, if needed, and microsurgical-guided testicular biopsies are performed by carefully removing enlarged tubules using microsurgical forceps. If enlarged tubules are not seen, then any tubule different than the remaining ones in size is excised. If all tubules are identical in appearance, random micro-biopsies are performed at each testicular pole. The excised testicular tissue specimens are placed into the outer-well Petri dish containing sperm media. Specimens are washed grossly to remove blood clots and are sent to the laboratory for processing and search for sperm (Figure-3). Albuginea and scrotal layers are closed using non-absorbable and absorbable sutures, respectively.

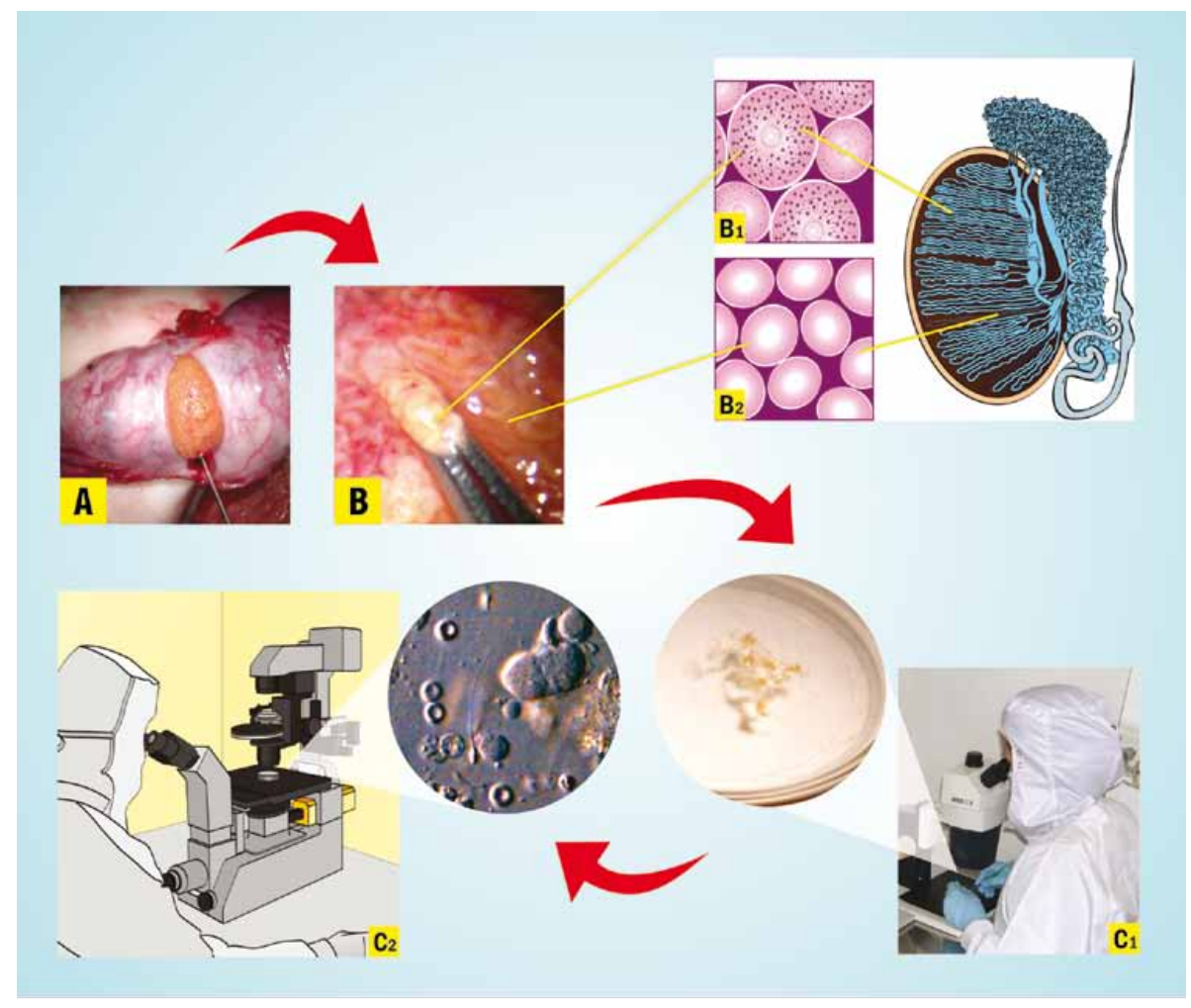

ANDROFERT 2011

Figure 3 - Microsurgical Testicular Sperm Extraction (Micro-TESE). Operating microscope and microsurgical technique are used throughout the procedures. After testis exteriorization, a single and large incision is made in an avascular area of the albuginea to expose testicular parenchyma (A). Microdissection of seminiferous tubules is carried out to identify and remove large tubules that are most likely to contain germ cells and active spermatogenesis. A photograph taken at x40-magnification indicates enlarged and non-enlarged seminiferous tubules (B). Enlarged tubules may contain active spermatogenesis, as illustrated in the transversal section of a histopathology specimen (B1). Non-enlarged tubules are more likely to contain no active spermatogenesis (B2). Excised testicular specimens are sent to the laboratory for processing and examination. Seminiferous tubules are mechanically dispersed using needled-tuberculin syringes (C1). Testicular homogenates are microscopically examined to confirm the presence of spermatozoa (C2). 


\section{Conventional Testicular Sperm Extraction (TESE)}

Extraction of testicular parenchyma for sperm search and their use in association with ICSI was first described by Devroey et al. in 1995 (3). For conventional TESE, a standard open surgical biopsy technique is used to retrieve sperm without the aid of optical magnification. TESE can be performed under either local anesthesia with or without intravenous sedation or epidural anesthesia, and it is often carried out using the 'window' technique. Briefly, a transverse $2 \mathrm{~cm}$ incision is made through the anterior scrotal skin, dartos and tunica vaginalis. A small self-retaining eyelid retractor is placed to improve exposure of the tunica albuginea, since the testis is not exteriorized. The albuginea is incised for approximately $1 \mathrm{~cm}$. Gentle pressure is made onto the testis to extrude testicular parenchyma. A small fragment (approximately $5 \times 5 \mathrm{~mm}$ ) is excised with sharp scissors and placed promptly in sperm culture media (Figure-2B). A single specimen or multiple specimens can be extracted from the same incision. Alternatively, individual albuginea incisions can be made onto the upper, middle and lower testicular poles to extract multiple biopsy specimens. Testicular specimens are sent to the laboratory for processing and immediate microscopic examination (Figure-2C). Albuginea is closed using non-absorbable sutures.

\section{Sperm Retrieval Postoperative Care and Compli- cations}

Percutaneous and open (microsurgical or conventional) sperm retrievals are usually carried out on an outpatient basis. Patients often resume their normal activities on the following day after percutaneous retrievals, and after 2-3 days following open surgical retrievals. Scrotal ice packing and scrotal supporter is recommended to decrease local edema and alleviate pain. Patients are counseled to restrain from ejaculation and strenuous physical activity for approximately 7-10 days. Oral analgesics and anti-inflammatory agents are usually prescribed because of the complaint of pain and scrotal swelling ranges from minimal to moderate.

The incidence of post-sperm retrieval complications ranges from $0-70 \%$ and include persistent pain, swelling, infection, hydrocele and hematoma (18-21). Complication rates vary according to the sperm retrieval technique and to a lesser extent to the type of azoospermia. PESA complications are usually of minimal morbidity although fibrosis at the aspiration site is often seen (6). Intratesticular hematoma has been observed in most patients undergoing TESE with single or multiple biopsies based on ultrasounds results performed after surgery, but they often resolve spontaneously without compromising testicular function (20). However, it has been reported that large-volume conventional TESE is associated with a higher risk of transient or even permanent decrease in serum testosterone levels due to testicular devascularization $(19,22)$. The incidence of complications following micro-TESE is lower than conventional TESE $(17,19,21,23)$. Using micro-TESE, identification of testicular vessels under the tunica albuginea is made prior to the placement of an incision into the testis. The use of optical magnification and microsurgical technique allow the preservation of intratesticular blood supply as well as the identification of tubules more likely to harbor sperm production (19). However, a significant decrease on serum testosterone has been documented following micro-TESE in men who already have diminished androgen production, such as KS patients (18). Nonetheless, testosterone levels return to the pre-surgical values in most individuals in a 12-month follow-up period. It is recommended that sperm retrievals should be performed by surgeons who have training in the procedures because of the potential serious postoperative complications (22).

\section{LABORATORY PROCESSING OF SURGI- CALLY-RETRIEVED SPERM}

Surgically retrieved-spermatozoa are often compromised in quality, particularly in the cases of NOA and after freezing and thawing (24). Therefore, great caution should be applied during processing of such specimens. In this regard, the laboratory has a crucial role not only to ease the sperm search and the selection of the best quality spermatozoa for ICSI but also to maintain the optimal sperm fertilizing ability (25). In order to achieve their goals, laboratory personnel should: i) receive the best quality surgically-retrieved specimen possible, with minimal or no 
contaminants such as red blood cells and noxious microorganisms; ii) minimize iatrogenic cellular damage during sperm processing by mastering technical skills and controlling factors, such as centrifugation force and duration, exposure to ultraviolet light and temperature variation, laboratory air quality conditions, dilution and washing steps, quality of reagents, culture media and disposable materials; and iii) improve sperm fertilizing potential, if possible, by using stimulants (26) or selecting viable sperm for ICSI when only immotile spermatozoa is available (27).

Testicular specimens are processed by either mechanical mincing/shredding the whole tissue or enzymatic digestion. The aim of tissue processing is to facilitate sperm search, and both mechanical and enzymatic techniques yield similar results $(28,29)$. After processing, epididymal and testicular spermatozoa can be either used for ICSI or cryopreserved. Cryopreserved specimens are thawed and processed using the principles already described (30). Methods for selecting viable sperm for ICSI are currently available in cases immotile spermatozoa only are obtained after processing $(27,31)$.

\section{SPERM RETRIEVAL SUCCESS RATES AND INTRACYTOPLASMIC SPERM INJECTION OUTCOMES}

The best sperm retrieval technique for men with either OA or NOA is yet to be determined. Randomized controlled trials are lacking to compare the efficiency of the available methods and current recommendations are based upon cumulative evidence provided by descriptive, observational and controlled studies $(7,9,22,23,32)$. The advantages and limitations of sperm retrieval techniques are shown in Table- 2 .

\section{Obstructive Azoospermia}

Both percutaneous and microsurgical methods yields high success rates, in the range of 90$100 \%$, for obtaining spermatozoa in OA $(6,9,33)$. It is recommended to commence aspiration at the corpus epididymis, and proceed to the caput if ne- cessary, since aspirates from the cauda are usually rich in poor quality senescent spermatozoa, debris and macrophages (6). Epididymal sperm retrieval may fail in certain cases of epididymal fibrosis caused by multiple previous retrieval attempts or post-infection. In such cases, sperm retrieval can be attempted in the contralateral epididymis or in the testis (6). In a recent series of 142 men with OA, cumulative successful retrieval rate after percutaneous aspirations was $97.9 \%$ (33). Retrievals succeeded in approximately $86 \%$ of cases using PESA alone, although multiple epididymal punctures were required in nearly half of procedures. Rescue TESA yielded approximately $90 \%$ success rate in cases of failed PESA (33). In the aforementioned study, sperm retrieval success rates using percutaneous techniques were similar regardless of the cause of obstruction being vasectomy, CBAVD and post-infectious etiology categories.

The choice of sperm retrieval by either percutaneous or open surgery from either the testis or epididymis should be based upon local preferences and expertise since there is no evidence that the site or method of sperm retrieval affects outcome of ICSI for patients with OA $(6,9,24,32)$. Moreover, neither the cause of obstruction nor the use of fresh or frozen-thawed epididymal/testicular sperm seems to have any significant effect on the success of assisted reproduction with regard to fertilization, pregnancy, or miscarriage rates (33-35). ICSI provides fertilization rates of $45-75 \%$ per injected oocyte when epididymal or testicular spermatozoa from men with OA are used. In such cases, clinical pregnancy and live birth rates reported in the recent literature range from $26-57 \%$ and $18-55 \%$, respectively (24,35-38) (Table-3).

\section{Non-obstructive Azoospermia}

Recent series with NOA report overall successful retrieval rates (SRR) ranging from $30-50 \%$. Testicular sperm have been obtained in all etiology categories of cryptorchidism, orchitis, genetic, radio-/chemotherapy and idiopathic $(5,6,10,17,18,21,23)$. Efficiency of sperm retrieval in NOA males varies according to the method 
Table 2 - Advantages and Disadvantages of Sperm Retrieval Techniques for Assisted Reproduction.

\begin{tabular}{|c|c|c|}
\hline & Advantages & Disadvantages \\
\hline PESA & $\begin{array}{c}\text { Fast and low cost } \\
\text { Minimal morbidity, repeatable } \\
\text { No microsurgical expertise required } \\
\text { Few instruments and materials } \\
\text { No surgical exploration }\end{array}$ & $\begin{array}{c}\text { Few sperm retrieved } \\
\text { Cryopreservation limited } \\
\text { Fibrosis and obstruction at } \\
\text { aspiration site } \\
\text { Risk of hematoma/spermatocele }\end{array}$ \\
\hline MESA & $\begin{array}{l}\text { Large number of sperm retrieved } \\
\text { Excellent chance of sperm cryopreservation } \\
\text { Reduced risk of hematoma } \\
\text { Reconstruction possible } \\
{ }^{1}\end{array}$ & $\begin{array}{l}\text { Surgical exploration required } \\
\text { Increased cost and time- } \\
\text { demanding } \\
\text { Microsurgical instruments and } \\
\text { expertise required } \\
\text { Postoperative discomfort }\end{array}$ \\
\hline TESA & $\begin{array}{c}\text { Fast and low cost } \\
\text { Repeatable } \\
\text { No microsurgical expertise required } \\
\text { Few instruments and materials } \\
\text { No surgical exploration } \\
\text { Minimal/mild postoperative discomfort }\end{array}$ & $\begin{array}{l}\text { Relatively low success rate in } \\
\text { NOA } \\
\text { Few sperm retrieved in NOA } \\
\text { Cryopreservation limited } \\
\text { Risk of hematoma/testicular } \\
\text { atrophy }\end{array}$ \\
\hline TESE & $\begin{array}{c}\text { No microsurgical expertise required } \\
\text { Fast and repeatable }\end{array}$ & $\begin{array}{c}\text { Relatively low success rate in } \\
\text { NOA } \\
\text { Relatively few sperm retrieved in } \\
\text { NOA } \\
\text { Risk of testicular atrophy (with } \\
\text { multiple biopsies) } \\
\text { Postoperative discomfort }\end{array}$ \\
\hline Micro-TESE & $\begin{array}{c}\text { Higher success rates in } \mathrm{NOA}^{2} \\
\text { Larger number of sperm retrieved }{ }^{2} \\
\text { Relatively higher chance of sperm cryopreservation }{ }^{2} \\
\text { Low risk of complications }\end{array}$ & $\begin{array}{l}\text { Surgical exploration required } \\
\text { Increased cost and time- } \\
\quad \text { demanding } \\
\text { Microsurgical instruments and } \\
\text { expertise required } \\
\text { Postoperative discomfort }\end{array}$ \\
\hline
\end{tabular}

PESA: percutaneous epididymal sperm aspiration; MESA: microsurgical epididymal sperm Aspiration; TESA: percutaneous testicular sperm aspiration; TESE: conventional testicular sperm extraction; micro-TESE: microsurgical testicular sperm extraction.

1 - In cases of vasectomy; 2 - Compared to TESA and TESE in NOA

of sperm collection. TESA retrieval rates range from $10-30 \%(22,23,39-41)$, except in the favorable cases of previous successful TESA or testicular histopathology showing hypospermatogenesis. In such cases, TESA SRR range from $70-100 \%$ $(6,10)$. In a recent systematic review the mean re- ported SRR for TESE was 49.5\% (23). TESE with multiple biopsies resulted in higher SRR than fine-needle aspiration (TEFNA), a variation of TESA, especially in cases of Sertoli-cell-only (SCO) and maturation arrest (23). Retrieval rates ranging from $35 \%$ to $77 \%$ have been reported for micro-TESE 
Table 3 - Sperm Retrieval (SR) Success Rates and Intracytoplasmic Sperm Injection Outcomes (ICSI) Using SurgicallyRetrieved Spermatozoa from Men with Obstructive and Nonobstructive Azoospermia.

\section{Obstructive Azoospermia}

Nonobstructive

Azoospermia

SR Success Rates by Method; Mean (Range)

Percutaneous

Microsurgical

ICSI Outcomes; Mean (Range)

2PN Fertilization Rate

Clinical Pregnancy Rate

Live Birth Rate
$90 \%(80-100 \%)$

$90 \%(80-100 \%)$

$60 \%(45-75 \%)$

$50 \%(26-57 \%)$

$35 \%(18-55 \%)$
$35 \%(10-100 \%)$

$50 \%(20-100 \%)$

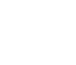

(17-19,21,23,42,43). Current evidence suggests that micro-TESE performs better than conventional TESE or TESA in cases of maturation arrest and SCO, where tubules containing active focus of spermatogenesis can be identified $(6,10,42)$. Moreover, tissue removal in micro-TESE is often 50 to 70 -fold less than conventional TESE $(17,43)$, and the small amount of tissue extracted facilitates sperm processing.

Preoperative predictive factors for sperm retrieval in NOA include testicular histopathology, serum FSH levels and genetic testing results $(6,12,13,44)$. Although not absolute, testicular histology is still considered the best predictor for successful sperm retrieval in NOA. Retrieval rates of approximately $85 \%$ are obtained in men presenting with hypospermatogenesis while testicular sperm can be collected in only $6-25 \%$ of men with the unfavorable histopathology pattern of germinal aplasia $(6,10,42,44)$. Follicle-stimulating hormone levels have also been used as a marker of testicular reserve, but it has been recently demonstrated that FSH levels in NOA men are not predictive of SRR $(45,46)$. Although FSH levels reflect the global spermatogenic function, adequate feedback control from germ cells and Sertoli cells is usually intact in cases of diffuse maturation arrest despite the absence of sperm production (45). In genetic-related NOA, such as Y-chromosome infertility and Klinefelter syndrome (KS), pregnancies may be achieved by ICSI in males with retrievable testicular sperm $(13,18)$. Testicular sperm can be found in approximately $70 \%$ of men with partial or complete AZFc deletion. In contrast, the chance of finding sperm in azoospermic men with complete AZFa or AZFb deletions is unlikely $(8,12,13)$. In case a successful pregnancy is induced by men with Y-chromosome azoospermia, the male offspring will harbor the same deletion as their father, with a high risk of male infertility. In NOA men with $\mathrm{KS}$, sperm are found in approximately $50 \%$ of cases on testicular exploration and pregnancy rates by ICSI range from $30 \%$ to $50 \%$ (18). It has been demonstrated that children that have been born have normal karyotype because germ cells in men with KS are euploid, 46,XY, and thus can form normal, haploid gametes (47).

The importance of surgical and medical treatment prior to sperm retrieval in NOA men has been recently highlighted. It has been suggested that treatment of clinical varicoceles prior to 
sperm retrieval significantly increased the chance of testicular sperm collection by micro-TESE in NOA individuals with clinical varicoceles (48). In this aforementioned study, SRR rates were 53\% and $30 \%$ in the treated and untreated men, respectively (odds-ratio [OR]: $2.63 ; 95 \%$ confidence interval $[\mathrm{CI}]$ of $1.05-6.60, \mathrm{p}=0.03)$. Medical therapy (aromatase inhibitors, clomiphene or human chorionic gonadotropin) prior to micro-TESE was also shown to enhance sperm retrieval success rates in Klinefelter syndrome men who responded to medication by increasing serum testosterone to more than $100 \mathrm{ng} / \mathrm{dL}$ from baseline (49).

Sperm retrieval may be performed either the day before or on the same day as the oocyte retrieval. Fresh sperm is preferable for ICSI because frozen-thawed surgically-retrieved sperm from NOA men have significantly impaired reproductive potential $(50,51)$. From the limited data available, it is suggested that the sperm retrieval technique itself has no impact on ICSI success rates in NOA (23). However, ICSI provides lower fertilization rates per injected oocyte as well as clinical pregnancy and delivery rates when testicular spermatozoa from men with NOA are used in comparison to ejaculated sperm or epididymal/ testicular sperm from men with OA $(24,38,52)$ (Table-3). Such differences may be explained by the fact that testicular spermatozoa from men with severely impaired spermatogenesis have a higher tendency to carry deficiencies such as the ones related to the centrioles and genetic material, which ultimately affect the capability of the male gamete to activate the egg and trigger the formation and development of a normal zygote and a viable embryo (53).

The risks of congenital malformations, infertility and other diseases in children conceived by using surgically-retrieved sperm for ICSI are still poorly determined. Assisted reproduction techniques (IVF or ICSI), as a whole, are associated with multiple gestation and elevated risk of congenital abnormalities compared to the rate of malformations in children conceived naturally (1-4\% rate) (54). ICSI, in particular, carries an increased risk of endocrine abnormalities as well as epigenetic imprinting effects $(54,55)$. Although the absolute risk of any of these conditions re- mains low (54-56), current data is limited and it is therefore recommended that well-defined groups of ICSI with ejaculated sperm, ICSI with epididymal sperm and ICSI with testicular sperm, and a control group of naturally conceived children are closely followed-up.

\section{CONFLICT OF INTEREST}

None declared.

\section{REFERENCES}

1. Palermo G, Joris H, Devroey P, Van Steirteghem AC: Pregnancies after intracytoplasmic injection of single spermatozoon into an oocyte. Lancet. 1992; 340: 17-8.

2. Silber SJ, Nagy ZP, Liu J, Godoy H, Devroey P, Van Steirteghem AC: Conventional in-vitro fertilization versus intracytoplasmic sperm injection for patients requiring microsurgical sperm aspiration. Hum Reprod. 1994; 9: 1705-9.

3. Devroey P, Liu J, Nagy Z, Goossens A, Tournaye H, Camus M, et al.: Pregnancies after testicular sperm extraction and intracytoplasmic sperm injection in non-obstructive azoospermia. Hum Reprod. 1995; 10: 1457-60.

4. Esteves SC, Miyaoka R, Agarwal A: An update on the clinical assessment of the infertile male. Clinics (Sao Paulo). 2011; 66: 691-700.

5. Schlegel PN: Causes of azoospermia and their management. Reprod Fertil Dev. 2004; 16: 561-72.

6. Esteves SC, Agarwal A. Sperm retrieval techniques. In: Gardner DK, Rizk BRMB, Falcone T. Human assisted reproductive technology: future trends in laboratory and clinical practice. Cambridge: Cambridge University Press, 2011, pp. 41-53.

7. Male Infertility Best Practice Policy Committee of the American Urological Association; Practice Committee of the American Society for Reproductive Medicine. Report on evaluation of the azoospermic male. Fertil Steril. 2006; 86(Suppl 1): S210-5.

8. Esteves SC, Agarwal A: Novel concepts in male infertility. Int Braz J Urol. 2011; 37: 5-15.

9. American Urological Association Education and Research, Inc. The management of obstructive azoospermia: AUA best practice statement. Linthicum (MD): American Urological Association Education and Research, Inc.; 2010. pp. 22 [cited May 19th, 2011]. Available at http://www.auanet.org/content/media/obstructiveazoospermial2010.pdf 
10. Esteves SC, Verza S, Prudencio C, Seol B: Sperm retrieval rates (SRR) in nonobstructive azoospermia (NOA) are related to testicular histopathology results but not to the etiology of azoospermia. Fertil Steril. 2010; 94 (Suppl): S132.

11. De Braekeleer M, Dao TN: Cytogenetic studies in male infertility: a review. Hum Reprod. 1991; 6: 245-50.

12. Brandell RA, Mielnik A, Liotta D, Ye Z, Veeck LL, Palermo GD, et al.: AZFb deletions predict the absence of spermatozoa with testicular sperm extraction: preliminary report of a prognostic genetic test. Hum Reprod. 1998; 13: 2812-5.

13. Hopps CV, Mielnik A, Goldstein M, Palermo GD, Rosenwaks Z, Schlegel PN: Detection of sperm in men with $\mathrm{Y}$ chromosome microdeletions of the $\mathrm{AZFa}, \mathrm{AZFb}$ and $\mathrm{AZFc}$ regions. Hum Reprod. 2003; 18: 1660-5.

14. Craft I, Shrivastav P: Treatment of male infertility. Lancet. 1994; 344: 191-2.

15. Lewin A, Weiss DB, Friedler S, Ben-Shachar I, Porat-Katz A, Meirow D, et al.: Delivery following intracytoplasmic injection of mature sperm cells recovered by testicular fine needle aspiration in a case of hypergonadotropic azoospermia due to maturation arrest. Hum Reprod. 1996; 11: 769-71.

16. Temple-Smith PD, Southwick GJ, Yates CA, Trounson AO, de Kretser DM: Human pregnancy by in vitro fertilization (IVF) using sperm aspirated from the epididymis. J In Vitro Fert Embryo Transf. 1985; 2: 119-22.

17. Schlegel PN: Testicular sperm extraction: microdissection improves sperm yield with minimal tissue excision. Hum Reprod. 1999; 14: 131-5.

18. Schiff JD, Palermo GD, Veeck LL, Goldstein M, Rosenwaks Z, Schlegel PN: Success of testicular sperm extraction [corrected] and intracytoplasmic sperm injection in men with Klinefelter syndrome. J Clin Endocrinol Metab. 2005; 90: 6263-7. Erratum in: J Clin Endocrinol Metab. 2006; 91: 4027.

19. Ramasamy R, Yagan N, Schlegel PN: Structural and functional changes to the testis after conventional versus microdissection testicular sperm extraction. Urology. 2005; 65: 1190-4.

20. Carpi A, Menchini Fabris FG, Palego P, Di Coscio G, Romani R, et al.: Fine-needle and large-needle percutaneous aspiration biopsy of testicles in men with nonobstructive azoospermia: safety and diagnostic performance. Fertil Steril. 2005; 83: 1029-33.

21. Turunc T, Gul U, Haydardedeoglu B, Bal N, Kuzgunbay B, Peskircioglu L, et al.: Conventional tes- ticular sperm extraction combined with the microdissection technique in nonobstructive azoospermic patients: a prospective comparative study. Fertil Steril. 2010; 94: 2157-60.

22. Carpi A, Sabanegh E, Mechanick J: Controversies in the management of nonobstructive azoospermia. Fertil Steril. 2009; 91: 963-70.

23. Donoso P, Tournaye H, Devroey P: Which is the best sperm retrieval technique for non-obstructive azoospermia? A systematic review. Hum Reprod Update. 2007; 13: 539-49.

24. Verza S Jr, Esteves SC: Sperm defect severity rather than sperm Source is associated with lower fertilization rates after intracytoplasmic sperm injection. Int Braz J Urol. 2008; 34: 49-56.

25. Rhoden EL, Soares JB, Esteves SC: O que o laboratório pode fazer pelo espermatozóide. II Consenso Brasileiro de Infertilidade Masculina. Int Braz J Urol. 2003 (Suppl); 29: 50-5.

26. Kovacic B, Vlaisavljevic V, Reljic M: Clinical use of pentoxifylline for activation of immotile testicular sperm before ICSI in patients with azoospermia. J Androl. 2006; 27: 45-52.

27. Esteves SC, Sharma RK, Thomas AJ Jr, Agarwal A: Suitability of the hypo-osmotic swelling test for assessing the viability of cryopreserved sperm. Fertil Steril. 1996; 66: 798-804.

28. Verheyen G, De Croo I, Tournaye H, Pletincx I, Devroey $\mathrm{P}$, van Steirteghem AC: Comparison of four mechanical methods to retrieve spermatozoa from testicular tissue. Hum Reprod. 1995; 10: 2956-9.

29. Baukloh V; German Society for Human Reproductive Biology: Retrospective multicentre study on mechanical and enzymatic preparation of fresh and cryopreserved testicular biopsies. Hum Reprod. 2002; 17: 1788-94.

30. Verza Jr S, Feijo CM, Esteves SC: Resistance of human spermatozoa to cryoinjury in repeated cycles of thaw-refreezing. Int Braz J Urol. 2009; 35: 581-90; discussion 591.

31. Soares JB, Glina S, Antunes N Jr, Wonchockier R, Galuppo AG, Mizrahi FE: Sperm tail flexibility test: a simple test for selecting viable spermatozoa for intracytoplasmic sperm injection from semen samples without motile spermatozoa. Rev Hosp Clin Fac Med Sao Paulo. 2003; 58: 250-3.

32. Van Peperstraten A, Proctor ML, Johnson NP, Philipson G: Techniques for surgical retrieval of sperm prior to ICSI for azoospermia. Cochrane Database Syst Rev. 2006; 3: CD002807. Update in: Cochrane Database Syst Rev. 2008;(2):CD002807. 
33. Esteves SC, Verza S, Prudencio C, Seol B: Success of percutaneous sperm retrieval and intracytoplasmic sperm injection (ICSI) in obstructive azoospermic (OA) men according to the cause of obstruction. Fertil Steril. 2010; 94 (Suppl): S233.

34. Kamal A, Fahmy I, Mansour R, Serour G, Aboulghar M, Ramos L, et al.: Does the outcome of ICSI in cases of obstructive azoospermia depend on the origin of the retrieved spermatozoa or the cause of obstruction? A comparative analysis. Fertil Steril. 2010; 94: 2135-40.

35. Nagy Z, Liu J, Cecile J, Silber S, Devroey P, Van Steirteghem A: Using ejaculated, fresh, and frozen-thawed epididymal and testicular spermatozoa gives rise to comparable results after intracytoplasmic sperm injection. Fertil Steril. 1995; 63: 808-15.

36. Tournaye H, Merdad T, Silber S, Joris H, Verheyen G, Devroey P, et al.: No differences in outcome after intracytoplasmic sperm injection with fresh or with frozen-thawed epididymal spermatozoa. Hum Reprod. 1999; 14: 90-5.

37. Habermann H, Seo R, Cieslak J, Niederberger C, Prins GS, Ross L: In vitro fertilization outcomes after intracytoplasmic sperm injection with fresh or frozen-thawed testicular spermatozoa. Fertil Steril. 2000; 73: 955-60.

38. Nicopoullos JD, Gilling-Smith C, Almeida PA, Norman-Taylor J, Grace I, Ramsay JW: Use of surgical sperm retrieval in azoospermic men: a metaanalysis. Fertil Steril. 2004; 82: 691-701.

39. Hauser R, Yogev L, Paz G, Yavetz H, Azem F, Lessing JB, et al.: Comparison of efficacy of two techniques for testicular sperm retrieval in nonobstructive azoospermia: multifocal testicular sperm extraction versus multifocal testicular sperm aspiration. J Androl. 2006; 27: 28-33.

40. Friedler S, Raziel A, Strassburger D, Soffer Y, Komarovsky D, Ron-El R: Testicular sperm retrieval by percutaneous fine needle sperm aspiration compared with testicular sperm extraction by open biopsy in men with non-obstructive azoospermia. Hum Reprod. 1997; 12: 1488-93.

41. Ezeh UI, Moore HD, Cooke ID: A prospective study of multiple needle biopsies versus a single open biopsy for testicular sperm extraction in men with non-obstructive azoospermia. Hum Reprod. 1998; 13: 3075-80.

42. Okada H, Dobashi M, Yamazaki T, Hara I, Fujisawa $\mathrm{M}$, Arakawa S, et al.: Conventional versus microdissection testicular sperm extraction for nonobstructive azoospermia. J Urol. 2002; 168: 1063-7.
43. Amer M, Ateyah A, Hany R, Zohdy W: Prospective comparative study between microsurgical and conventional testicular sperm extraction in non-obstructive azoospermia: follow-up by serial ultrasound examinations. Hum Reprod. 2000; 15: 653-6.

44. Tournaye H, Verheyen G, Nagy P, Ubaldi F, Goossens A, Silber S, et al.: Are there any predictive factors for successful testicular sperm recovery in azoospermic patients? Hum Reprod. 1997; 12: 80-6.

45. Hung AJ, King P, Schlegel PN: Uniform testicular maturation arrest: a unique subset of men with nonobstructive azoospermia. J Urol. 2007; 178: 60812; discussion 612.

46. Ramasamy R, Lin K, Gosden LV, Rosenwaks Z, Palermo GD, Schlegel PN: High serum FSH levels in men with nonobstructive azoospermia does not affect success of microdissection testicular sperm extraction. Fertil Steril. 2009; 92: 590-3.

47. Sciurano RB, Luna Hisano CV, Rahn MI, Brugo Olmedo S, Rey Valzacchi G, Coco R, et al.: Focal spermatogenesis originates in euploid germ cells in classical Klinefelter patients. Hum Reprod. 2009; 24: 2353-60.

48. Inci K, Hascicek M, Kara O, Dikmen AV, Gürgan $T$, Ergen A: Sperm retrieval and intracytoplasmic sperm injection in men with nonobstructive azoospermia, and treated and untreated varicocele. $\mathrm{J}$ Urol. 2009; 182: 1500-5.

49. Ramasamy R, Ricci JA, Palermo GD, Gosden LV, Rosenwaks Z, Schlegel PN: Successful fertility treatment for Klinefelter's syndrome. J Urol. 2009; 182: 1108-13.

50. Fischer R, Baukloh V, Naether OG, Schulze W, Salzbrunn A, Benson DM: Pregnancy after intracytoplasmic sperm injection of spermatozoa extracted from frozen-thawed testicular biopsy. Hum Reprod. 1996; 11: 2197-9.

51. Schlegel PN, Liotta D, Hariprashad J, Veeck LL: Fresh testicular sperm from men with nonobstructive azoospermia works best for ICSI. Urology. 2004; 64: 1069-71.

52. Prudencio C, Seol B, Esteves SC: Reproductive potential of azoospermic men undergoing intracytoplasmic sperm injection is dependent on the type of azoospermia. Fertil Steril. 2010; 94 (Suppl): S232-3.

53. Tesarik J: Paternal effects on cell division in the human preimplantation embryo. Reprod Biomed Online. 2005; 10: 370-5.

54. Alukal JP, Lamb DJ: Intracytoplasmic sperm injection (ICSI)--what are the risks? Urol Clin North Am. 2008; 35: 277-88. 
55. Knoester M, Helmerhorst FM, Vandenbroucke JP, van der Westerlaken LA, Walther FJ, Veen S, et al.: Cognitive development of singletons born after intracytoplasmic sperm injection compared with in vitro fertilization and natural conception. Fertil Steril. 2008; 90: 289-96.
56. Hansen M, Bower C, Milne E, de Klerk N, Kurinczuk JJ: Assisted reproductive technologies and the risk of birth defects--a systematic review. Hum Reprod. 2005; 20: 328-38.
Submitted for publication:

June 22, 2010

Accepted after revision:

July 15, 2011

\section{Correspondence address:}

Dr. Sandro Esteves

ANDROFERT

Av. Dr. Heitor Penteado, 1464

Campinas, São Paulo, 13075-460, Brazil

FAX: +55 19 3294-6992

E-mail: s.esteves@androfert.com.br 